\title{
On the locus of Prym curves where the Prym-canonical map is not an embedding
}

Ciro Ciliberto, Thomas Dedieu, Concettina Galati and Andreas Leopold Knutsen

\begin{abstract}
We prove that the locus of Prym curves $(C, \eta)$ of genus $g \geq 5$ for which the Prymcanonical system $\left|\omega_{C}(\eta)\right|$ is base point free but the Prym-canonical map is not an embedding is irreducible and unirational of dimension $2 g+1$.
\end{abstract}

\section{Introduction}

Let $g \geqslant 2$ and $\mathcal{R}_{g}$ be the moduli space of Prym curves, that is, of pairs $(C, \eta)$, with $C$ a smooth complex projective genus $g$ curve and $\eta$ a non-zero 2-torsion point of $\mathrm{Pic}^{0}(C)$. It is well-known that $\mathcal{R}_{g}$ is irreducible of dimension $3 g-3$ and that the natural forgetful map $\mathcal{R}_{g} \rightarrow \mathcal{M}_{g}$, where $\mathcal{M}_{g}$ denotes the moduli space of smooth genus $g$ curves, is finite of degree $2^{2 g}-1$. The complete linear system $\left|\omega_{C}(\eta)\right|$ is of dimension $g-2$ and it is base point free unless $C$ is hyperelliptic and $\eta \simeq \mathcal{O}_{C}(p-q)$, with $p$ and $q$ ramification points of the $g_{2}^{1}$ (cf. Lemma 2.1 below).

In this note we study the locally closed locus $\mathcal{R}_{g}^{0}$ in $\mathcal{R}_{g}$ of Prym curves $(C, \eta)$ such that the Prym-canonical system $\left|\omega_{C}(\eta)\right|$ is base point free but the morphism $C \rightarrow \mathbb{P}^{g-2}$ it defines (the so-called Prym-canonical map) is not an embedding. Note that $\mathcal{R}_{g}^{0}$ is clearly dense in $\mathcal{R}_{g}$ for $g \leqslant 4$. Our main result is the following:

Theorem 1.1. Let $g \geqslant 5$. The locus $\mathcal{R}_{g}^{0}$ is irreducible and unirational of dimension $2 g+1$ and lies in the tetragonal locus.

By the tetragonal locus $\mathcal{R}_{g, 4}^{1}$ in $\mathcal{R}_{g}$ we mean the inverse image via $\mathcal{R}_{g} \rightarrow \mathcal{M}_{g}$ of the tetragonal locus $\mathcal{M}_{g, 4}^{1}$ of $\mathcal{M}_{g}$.

We also show:

Proposition 1.2. For general $(C, \eta) \in \mathcal{R}_{g}^{0}, g \geqslant 5$, the Prym-canonical map is birational onto its image, and its image has precisely two nodes. 
Although we believe that these results are of independent interest, our main motivation for studying the locus $\mathcal{R}_{g}^{0}$ is that it naturally contains pairs $(C, \eta)$ where $C$ is a smooth curve lying on an Enriques surface $S$ such that

$$
\phi(C)=\min \left\{E \cdot C \mid E \in \operatorname{Pic}(S), E>0, E^{2}=0\right\}=2,
$$

and $\eta=\mathcal{O}_{C}\left(K_{S}\right)$, cf. Examples 5.1 and 5.2 and Remark 5.5, in which case the Prymcanonical map associated to $\eta$ is the restriction to $C$ of the map defined by the complete linear system $|C|$ on $S$. The locus $\mathcal{R}_{g}^{0}$ indeed naturally shows up in our recent work [6] concerning the moduli of smooth curves lying on an Enriques surface, in which we use the results in this note. Besides, we show in [6] that $\mathcal{R}_{g}^{0}$ is dominated by curves on Enriques surfaces for $5 \leqslant g \leqslant 8$.

The paper is organized as follows. Section 2 is devoted to recalling some preliminary results. The irreducibility and unirationality of $\mathcal{R}_{g}^{0}$ is proved in Section 3, whereas its dimension is computed in Section 4. We conclude with the proof of Proposition 1.2 together with the mentioned examples on Enriques surfaces.

Acknowledgments. The authors thank Gian Pietro Pirola and Alessandro Verra for useful conversations on the subject and acknowledge funding from MIUR Excellence Department Project CUP E83C180 00100006 (CC), project FOSICAV within the EU Horizon 2020 research and innovation programme under the Marie Skłodowska-Curie grant agreement n. 652782 (CC, ThD), GNSAGA of INDAM (CC, CG), Bergen Research Foundation (ThD, ALK) and grant n. 261756 of the Research Council of Norway (ALK).

\section{Preliminary results}

\subsection{A basic lemma on Prym curves}

The following is an immediate consequence of the Riemann-Roch theorem (see also $[8, \S 0.1]$ or $[12$, Pf. of Lemma 2.1]). We include the proof for the reader's convenience.

Lemma 2.1. Let $(C, \eta)$ be any Prym curve of genus $g \geqslant 3$. Then:

(i) $p$ is a base point of $\left|\omega_{C}(\eta)\right|$ if and only if $|p+\eta| \neq \varnothing$. This happens if and only if $C$ is hyperelliptic and $\eta \sim \mathcal{O}_{C}(p-q)$, with $p$ and $q$ ramification points of the $g_{2}^{1}$. In particular, $p$ and $q$ are the only base points;

(ii) if $\left|\omega_{C}(\eta)\right|$ is base point free, then it does not separate $p$ and $q$ (possibly infinitely near) if and only if $|p+q+\eta| \neq \varnothing$. This happens if and only if $C$ has a $g_{4}^{1}$ and $\eta \sim \mathcal{O}_{C}(p+q-x-y)$, where $2(p+q)$ and $2(x+y)$ are members of the $g_{4}^{1}$. In particular, also $x$ and $y$ are not separated by $\left|\omega_{C}(\eta)\right|$. 
Proof. We prove only (ii) and leave (i) to the reader. Assume that $\left|\omega_{C}(\eta)\right|$ is base point free. Then $p$ and $q$ are not separated by the linear system $\left|\omega_{C}(\eta)\right|$ if and only if $h^{0}\left(\omega_{C}(\eta)-p\right)=h^{0}\left(\omega_{C}(\eta)-p-q\right)$. By Riemann-Roch and Serre duality, this is equivalent to $h^{0}(\eta+p)+1=h^{0}(\eta+p+q)$. By $(\mathrm{i})$, we have $h^{0}(\eta+p)=0$, whence the latter condition is $h^{0}(\eta+p+q)=1$. This is equivalent to $h^{0}(\eta+p+q)>0$, because if $h^{0}(\eta+p+q)>1$, then we would have $h^{0}(\eta+p)>0$, a contradiction. This proves the first assertion.

We have $|p+q+\eta| \neq \varnothing$ if and only if $p+q+\eta \sim x+y$, for $x, y \in C$. This implies $2(p+q) \sim 2(x+y)$, whence $C$ has a $g_{4}^{1}$ with $2(p+q)$ and $2(x+y)$ as its members. Conversely, if $2(p+q)$ and $2(x+y)$ are distinct members of a $g_{4}^{1}$ on $C$, then $\eta:=$ $\mathcal{O}_{C}(p+q-x-y)$ is a 2 -torsion element of $\operatorname{Pic}^{0}(C)$ and satisfies the condition that $|p+q+\eta| \neq \varnothing$.

The lemma says in particular that the locus in $\mathcal{R}_{g}$ of pairs $(C, \eta)$ for which the Prym-canonical system $\left|\omega_{C}(\eta)\right|$ is not base-point free dominates the hyperelliptic locus via the forgetful map $\mathcal{R}_{g} \rightarrow \mathcal{M}_{g}$.

Recall that the tetragonal locus $\mathcal{R}_{g, 4}^{1}$ is irreducible of dimension $2 g+3$ if $g \geqslant 7$ and coincides with $\mathcal{R}_{g}$ if $g \leqslant 6$. Lemma 2.1 implies that $\mathcal{R}_{g}^{0} \subseteq \mathcal{R}_{g, 4}^{1}$, thus proving the last statement in Theorem 1.1.

The lemma also enables us to detect the locus $\mathcal{R}_{g}^{0, \mathrm{nb}}$ in $\mathcal{R}_{g}^{0}$ where the Prymcanonical morphism is not birational onto its image:

Corollary 2.2. Let $(C, \eta)$ be any Prym curve of genus $g \geqslant 4$ such that the Prym-canonical system $\left|\omega_{C}(\eta)\right|$ is base point free. If the Prym-canonical map is not birational onto its image, then it is of degree two onto a smooth elliptic curve.

The locus $\mathcal{R}_{g}^{0, \mathrm{nb}}$ is irreducible of dimension $2 g-2$ and dominates the bielliptic locus in $\mathcal{M}_{g}$. More precisely, $\mathcal{R}_{g}^{0, \mathrm{nb}}$ consists of pairs $(C, \eta)$, with $C$ bielliptic and $\eta:=\varphi^{*} \eta^{\prime}$, where $\varphi: C \rightarrow E$ is a bielliptic map and $\eta^{\prime}$ is a nontrivial 2-torsion element in $\operatorname{Pic}^{0}(E)$.

Proof. Let $(C, \eta)$ be as in the statement. Denote by $C^{\prime}$ the image of the Prymcanonical morphism $\varphi: C \rightarrow \mathbb{P}^{g-2}$. Let $\mu$ be the degree of $\varphi$ and $d$ the degree of $C^{\prime}$. Then $d \mu=2 g-2$ and, since $C^{\prime}$ is non-degenerate in $\mathbb{P}^{g-2}$, we must have $d \geqslant g-2$. Since $g \geqslant 4$, then $2 \leqslant \mu \leqslant 3$; moreover $\mu=3$ implies that $g=4$ and $\varphi$ maps $C$ three-toone to a conic. The latter case cannot happen: indeed, we would have $\omega_{C}(\eta)=2 \mathcal{L}$, where $|\mathcal{L}|$ is a $g_{3}^{1}$. Then $4 \mathcal{L}=2 \omega_{C}$. Since $\left|2 \omega_{C}\right|$ is cut out by quadrics on the canonical image of $C$ in $\mathbb{P}^{3}$, it follows that the only quadric containing the canonical model is a cone. Then $|\mathcal{L}|$ is the unique $g_{3}^{1}$ on $C$ and $2 \mathcal{L}=\omega_{C}$, thus $\eta$ is trivial, a contradiction.

Hence $\mu=2$, and then $d=g-1$, so that $C^{\prime}$ is a curve of almost minimal degree. It is easy to see, using the fact that $\left|\omega_{C}(\eta)\right|$ is complete, that $C^{\prime}$ is a smooth elliptic curve (alternatively, apply [5, Thm. 1.2]). Hence $C$ is bielliptic and any 
pair of points $p$ and $q$ identified by $\varphi$ satisfy $p+q \sim \varphi^{*}(r)$ for a point $r \in C^{\prime}$. Thus $2 p+2 q \sim \varphi^{*}(2 r)$ is a $g_{4}^{1}$. By Lemma 2.1(ii) we have $\eta \sim \mathcal{O}_{C}(p+q-x-y)$, where also $\varphi(x)=\varphi(y)$, whence $x+y \sim \varphi^{*}(z)$, for a $z \in C^{\prime}$. Hence, again by Lemma 2.1(ii), we have $\eta \sim p+q-x-y \sim \varphi^{*}(r-z)$ and $r-z$ is a nontrivial 2-torsion element in $\operatorname{Pic}^{0}\left(C^{\prime}\right)$, because $\varphi^{*}: \mathrm{Pic}^{0}\left(C^{\prime}\right) \rightarrow \operatorname{Pic}^{0}(C)$ is injective.

Conversely, if $C$ is a bielliptic curve, it admits at most finitely many double covers $\varphi: C \rightarrow E$ onto an elliptic curve (cf. e.g., [2]; in fact, for $g \geqslant 6$, it admits a unique such map), and for any such $\varphi$ and any nontrivial 2-torsion element $\eta^{\prime}$ in $\operatorname{Pic}^{0}(E)$, we have $\eta^{\prime} \sim r-z$, for $r, z \in E$. Letting $\varphi^{*}(r)=p+q$ and $\varphi^{*}(z)=x+y$, we see that $2(p+q) \sim 2(x+y)$ and $\eta=\varphi^{*} \eta^{\prime}$ satisfies the conditions of Lemma 2.1(ii).

We have therefore proved that $\mathcal{R}_{g}^{0, \mathrm{nb}}$ consists of pairs $(C, \eta)$, with $C$ bielliptic and $\eta:=\varphi^{*} \eta^{\prime}$, where $\varphi: C \rightarrow E$ is a bielliptic map and $\eta^{\prime}$ is a nontrivial 2-torsion element in $\operatorname{Pic}^{0}(E)$.

The statement about the dimension of $\mathcal{R}_{g}^{0, \mathrm{nb}}$ follows since the bielliptic locus has dimension $2 g-2$. To prove its irreducibility, consider the map $f: \mathcal{R}_{g}^{0, \mathrm{nb}} \rightarrow \mathcal{R}_{1}$ associating to $(C, \eta)$ the pair $\left(E, \eta^{\prime}\right)$ as above. We study the fibres of this map. Consider the following obvious cartesian diagram defining $\mathcal{H}$, where $U \subset \operatorname{Sym}^{2 g-2}(E)$ is the open subset consisting of reduced divisors:

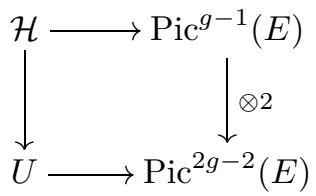

By Riemann's existence theorem, $\mathcal{H} / \operatorname{Aut}(E)$ is in one-to-one correspondence with the two-to-one covers of $E$ branched at $2 g-2$ points. Then the fibre of $f$ over $\left(E, \eta^{\prime}\right)$ is isomorphic to $\mathcal{H} / \operatorname{Aut}(E)$ by what we said above. Now note that $\mathcal{H}$ is irreducible, since it fibres over (an open subset of) $\mathrm{Pic}^{g-1}(E)$ with fibres that are projective spaces of dimension $2 g-3$. Hence also $\mathcal{H} / \operatorname{Aut}(E)$ is irreducible.

The irreducibility of $\mathcal{R}_{g}^{0, \mathrm{nb}}$ now follows from the irreducibility of $\mathcal{R}_{1}$. Actually $\mathcal{R}_{1}$ is irreducible and rational. To see this consider the irreducible family of elliptic curves $y^{2}=x(x-1)(x-\lambda)$, where $\lambda \in \mathbb{C} \backslash\{0,1\}$. The three non-trivial points of order two of the fibre $\mathcal{C}_{\lambda}$ over $\lambda$ may be identified with the points $(0,0),(1,0)$ and $(\lambda, 0)$. Moreover, the $j$-invariant of the fibres defines a six-to-one map $j: \mathbb{C} \backslash\{0,1\} \rightarrow \mathcal{M}_{1}$. Now consider on this family the two sections defined by the points $(0,0),(1,0)$ which stay fixed as $\lambda$ varies. It is an exercise to prove that the irreducible family of twomarked elliptic curves we obtain in this way is isomorphic to the moduli space of pairs $\left(C,\left(\eta_{1}, \eta_{2}\right)\right)$ where $C$ is a smooth elliptic curve and $\left(\eta_{1}, \eta_{2}\right)$ is an ordered pair of distinct non-trivial 2-torsion points of $\operatorname{Pic}^{0}(\mathrm{C})$. This moduli space is, in turn, isomorphic to the moduli space $\mathcal{M}_{1}^{(2)}$ of elliptic curves with a level 2 structure $[9$, 
Ex. 2.2.1]. Finally $\mathcal{M}_{1}^{(2)} \simeq \mathbb{C} \backslash\{0,1\}$ maps two-to-one dominantly to $\mathcal{R}_{1}$, via the $\operatorname{map}\left(C,\left(\eta_{1}, \eta_{2}\right)\right) \mapsto\left(C,\left(\eta_{1}+\eta_{2}\right)\right)$. This proves the statement.

\subsection{A result on linear systems on rational surfaces}

We will need the following:

Theorem 2.3. (cf. [1, Cor. (4.6)]) Let $X$ be a smooth projective rational surface and $\delta$ a non-negative integer. Let $\mathcal{L}$ be a complete linear system on $X$ such that:

(i) the general curve in $\mathcal{L}$ is smooth and irreducible;

(ii) the genus $p_{a}(\mathcal{L})$ of the general curve in $\mathcal{L}$ satisfies $p_{a}(\mathcal{L}) \geqslant \delta$;

(iii) $\operatorname{dim}(\mathcal{L})>3 \delta$;

(iv) if $p_{1}, \ldots, p_{\delta}$ are general points of $X$, there is an element $C$ of $\mathcal{L}$ singular at $p_{1}, \ldots, p_{\delta}$ such that for each irreducible component $C^{\prime}$ of $C$ one has $K_{X} \cdot C^{\prime}<0$.

Then, if $p_{1}, \ldots, p_{\delta}$ are general points of $X$ and $\mathcal{L}\left(p_{1}^{2}, \ldots, p_{\delta}^{2}\right)$ is the subsystem of $\mathcal{L}$ formed by the curves singular at $p_{1}, \ldots, p_{\delta}$, one has:

(a) the general curve in $\mathcal{L}\left(p_{1}^{2}, \ldots, p_{\delta}^{2}\right)$ is irreducible, has nodes at $p_{1}, \ldots, p_{\delta}$ and no other singularity;

(b) $\operatorname{dim}\left(\mathcal{L}\left(p_{1}^{2}, \ldots, p_{\delta}^{2}\right)\right)=\operatorname{dim}(\mathcal{L})-3 \delta$.

Proof. The proof of (a) is in [1]. As for (b), one has $\operatorname{dim}\left(\mathcal{L}\left(p_{1}^{2}, \ldots, p_{\delta}^{2}\right)\right)=$ $\operatorname{dim}(\mathcal{L})-3 \delta+\varepsilon$, with $\varepsilon \geqslant 0$. Consider the locally closed family of curves in $\mathcal{L}$ given by

$$
\mathcal{F}:=\bigcup_{p_{1}, \ldots, p_{\delta}} \mathcal{L}\left(p_{1}^{2}, \ldots, p_{\delta}^{2}\right),
$$

where the union is made by varying $p_{1}, \ldots, p_{\delta}$ among all the $\delta$-tuples of sufficiently general points of $X$. Of course

$$
\operatorname{dim}(\mathcal{F})=2 \delta+\operatorname{dim}\left(\mathcal{L}\left(p_{1}^{2}, \ldots, p_{\delta}^{2}\right)\right)=\operatorname{dim}(\mathcal{L})-\delta+\varepsilon .
$$

On the other hand, if $C$ is a general element in $\mathcal{F}$, it has nodes at $p_{1}, \ldots, p_{\delta}$ and no other singularity by (a), hence the Zariski tangent space to $\mathcal{F}$ at $C$ is the linear system $\mathcal{L}\left(p_{1}, \ldots, p_{\delta}\right)$ of curves in $\mathcal{L}$ containing $p_{1}, \ldots, p_{\delta}$. Since $p_{1}, \ldots, p_{\delta}$ are general, we have $\operatorname{dim}\left(\mathcal{L}\left(p_{1}, \ldots, p_{\delta}\right)\right)=\operatorname{dim}(\mathcal{L})-\delta$, which proves that $\varepsilon=0$.

\section{Irreducibility and unirationality of $\mathcal{R}_{g}^{0}$}

In this section we prove a first part of Theorem 1.1, namely:

Proposition 3.1. The locus $\mathcal{R}_{g}^{0}$ is irreducible and unirational for $g \geqslant 5$. 
The proof is inspired by the arguments in [1] and requires some preliminary considerations. In [1, Theorem (5.3)] the authors prove that some Hurwitz schemes $\mathcal{H}_{g, d}$ are unirational. Here we focus on the case $d=4$ and recall their construction.

Fix $g=2 h+\varepsilon \geqslant 3$, with $0 \leqslant \varepsilon \leqslant 1$. Then set $n=h+3+\varepsilon$ and

$$
\delta=\left(\begin{array}{c}
n-1 \\
2
\end{array}\right)-\left(\begin{array}{c}
n-4 \\
2
\end{array}\right)-g=h+2 \varepsilon .
$$

Fix now $p, p_{1}, \ldots, p_{\delta}$ general points in the projective plane and consider the linear system $\mathcal{L}_{n}\left(p^{n-4}, p_{1}^{2}, \ldots, p_{\delta}^{2}\right)$ of plane curves of degree $n$ having multiplicity at least $n-4$ at $p$ and multiplicity at least 2 at $p_{1}, \ldots, p_{\delta}$. As an application of Theorem 2.3 , in $\left[1\right.$, Cor. (4.7)] one proves that the dimension of $\mathcal{L}_{n}\left(p^{n-4}, p_{1}^{2}, \ldots, p_{\delta}^{2}\right)$ is the expected one, i.e.,

$$
\operatorname{dim}\left(\mathcal{L}_{n}\left(p^{n-4}, p_{1}^{2}, \ldots, p_{\delta}^{2}\right)\right)=\frac{n(n+3)}{2}-\frac{(n-4)(n-3)}{2}-3 \delta=2 h+9-\varepsilon,
$$

and the general curve $\Gamma$ in $\mathcal{L}_{n}\left(p^{n-4}, p_{1}^{2}, \ldots, p_{\delta}^{2}\right)$ is irreducible, has an ordinary $(n-$ 4 )-tuple point at $p$, nodes at $p_{1}, \ldots, p_{\delta}$, and no other singularity. The normalization $C$ of $\Gamma$ has genus $g$ and it has a $g_{4}^{1}$, which is the pull-back to $C$ of the linear series cut out on $\Gamma$ by the pencil of lines through $p$.

Consider then the locally closed family of curves

$$
\mathcal{H}:=\bigcup_{p_{1}, \ldots, p_{\delta}} \mathcal{L}_{n}\left(p^{n-4}, p_{1}^{2}, \ldots, p_{\delta}^{2}\right),
$$

where the union is made by varying $p_{1}, \ldots, p_{\delta}$ among all the $\delta$-tuples of sufficiently general points of the plane. Then $\mathcal{H}$ is clearly irreducible, rational, of dimension $\operatorname{dim}\left(\mathcal{L}_{n}\left(p^{n-4}, p_{1}^{2}, \ldots, p_{\delta}^{2}\right)\right)+2 \delta=4 h+9+3 \varepsilon$, and in [1] it is proved that the natural map $\mathcal{H}-\rightarrow \mathcal{M}_{g, 4}^{1}$ is dominant, so that $\mathcal{M}_{g, 4}^{1}$ is unirational.

Proof of Proposition 3.1. To prove our result, we slightly modify the above argument from [1]. Let us fix $g \geqslant 5, n, \delta$ as above. Let $p, p_{1}, \ldots, p_{\delta}$ be general points in the plane.

Claim 3.2. Consider the linear system $\mathcal{L}_{n-2}\left(p^{n-6}, p_{1}^{2}, \ldots, p_{\delta}^{2}\right)$ of plane curves of degree $n-2$, having a point of multiplicity at least $n-6$ at $p$, and singular at $p_{1}, \ldots, p_{\delta}$. Then the dimension of $\mathcal{L}_{n-2}\left(p^{n-6}, p_{1}^{2}, \ldots, p_{\delta}^{2}\right)$ is the expected one, i.e.,

$$
\operatorname{dim}\left(\mathcal{L}_{n-2}\left(p^{n-6}, p_{1}^{2}, \ldots, p_{\delta}^{2}\right)\right)=\frac{(n-2)(n+1)}{2}-\frac{(n-6)(n-5)}{2}-3 \delta=2 h-1-\varepsilon .
$$

Proof of Claim 3.2. Assume first $g=5$, which implies $(h, \varepsilon, n, \delta)=(2,1,6,4)$. Then one has $\mathcal{L}_{n-2}\left(p^{n-6}, p_{1}^{2}, \ldots, p_{\delta}^{2}\right)=\mathcal{L}_{4}\left(p_{1}^{2}, \ldots, p_{4}^{2}\right)$, which consists of all pairs of 
conics through $p_{1}, \ldots, p_{4}$, and has dimension 2 as desired. We can assume next that $g \geqslant 6$, hence $h \geqslant 3$ and $n \geqslant 6$.

Let $X$ be the blow-up of $\mathbb{P}^{2}$ at $p$. Note that the anticanonical system of $X$ is very ample. Consider the linear system $\mathcal{L}$ proper transform on $X$ of $\mathcal{L}_{n-2}\left(p^{n-6}\right)$. One checks that $X$ and $\mathcal{L}$ verify the hypotheses (i)-(iv) of Theorem 2.3. Indeed, (i) and (iv) are immediate, whereas (ii) and (iii) follow by standard computations and the fact that $h \geqslant 3$. Then the assertion follows by Theorem 2.3(b).

Next fix two distinct lines $r_{1}, r_{2}$ through $p$ and, for $1 \leqslant i \leqslant 2$, two distinct points $q_{i j}$, both different from $p$, on the line $r_{i}$, with $1 \leqslant j \leqslant 2$. Consider then the linear system $\mathcal{L}_{n}\left(p^{n-4}, p_{1}^{2}, \ldots, p_{\delta}^{2} ;\left[q_{11}, q_{12}, q_{21}, q_{22}\right]\right)$ consisting of all curves in $\mathcal{L}_{n}\left(p^{n-4}, p_{1}^{2}, \ldots, p_{\delta}^{2}\right)$ whose intersection multiplicity with $r_{i}$ at $q_{i j}$ is at least 2 , for $1 \leqslant i, j \leqslant 2$.

Claim 3.3. The linear system $\mathcal{L}_{n}\left(p^{n-4}, p_{1}^{2}, \ldots, p_{\delta}^{2} ;\left[q_{11}, q_{12}, q_{21}, q_{22}\right]\right)$ has the expected dimension, i.e.,

$$
\begin{aligned}
\operatorname{dim}\left(\mathcal{L}_{n}\left(p^{n-4}, p_{1}^{2}, \ldots, p_{\delta}^{2} ;\left[q_{11}, q_{12}, q_{21}, q_{22}\right]\right)\right) & =\frac{n(n+3)}{2}-\frac{(n-4)(n-3)}{2}-3 \delta-8 \\
& =2 h+1-\varepsilon
\end{aligned}
$$

and the general curve in $\mathcal{L}_{n}\left(p^{n-4}, p_{1}^{2}, \ldots, p_{\delta}^{2} ;\left[q_{11}, q_{12}, q_{21}, q_{22}\right]\right)$ is irreducible, has a point of multiplicity $n-4$ at $p$, has nodes at $p_{1}, \ldots, p_{\delta}$ and no other singularity, and is tangent at $r_{i}$ in $q_{i j}$, for $1 \leqslant i, j \leqslant 2$.

Proof of Claim 3.3. Let $X$ be the blow-up of the plane at $p$, at the points $q_{i, j}$ and at the infinitely near points to $q_{i j}$ along the line $r_{i}$, for $1 \leqslant i, j \leqslant 2$. Note that the anticanonical system of $X$ has a fixed part consisting of the strict transforms $R_{1}, R_{2}$ of $r_{1}, r_{2}$ plus the exceptional divisor $E$ over $p$, and a movable part consisting of the pull back to $X$ of the linear system of the lines in the plane.

Let $\mathcal{L}$ be the strict transform on $X$ of $\mathcal{L}_{n}\left(p^{n-4} ;\left[q_{11}, q_{12}, q_{21}, q_{22}\right]\right)$, the linear system of curves of degree $n$ with multiplicity at least $n-4$ at $p$ and whose intersection multiplicity with $r_{i}$ at $q_{i j}$ is at least 2 , for $1 \leqslant i, j \leqslant 2$. One has

$$
\operatorname{dim}(\mathcal{L})=\frac{n(n+3)}{2}-\frac{(n-4)(n-3)}{2}-8
$$

and an application of Bertini's theorem shows that the general curve in $\mathcal{L}$ is smooth and irreducible and its genus is

$$
p_{a}(\mathcal{L})=\left(\begin{array}{c}
n-1 \\
2
\end{array}\right)-\left(\begin{array}{c}
n-4 \\
2
\end{array}\right) \geqslant \delta .
$$

Moreover

$$
\operatorname{dim}(\mathcal{L})-3 \delta=2 h+1-\varepsilon>0 .
$$


Hence the linear system $\mathcal{L}\left(p_{1}^{2}, \ldots, p_{\delta}^{2}\right)$ of curves in $\mathcal{L}$ singular at $p_{1}, \ldots, p_{\delta}$ has dimension

$$
\operatorname{dim}\left(\mathcal{L}\left(p_{1}^{2}, \ldots, p_{\delta}^{2}\right)\right) \geqslant 2 h+1-\varepsilon .
$$

We claim that $\mathcal{L}\left(p_{1}^{2}, \ldots, p_{\delta}^{2}\right)$ does not have $R_{1}, R_{2}$ or $E$ in its fixed locus. Indeed, if $E$ is in this fixed locus, then clearly also $R_{1}$ and $R_{2}$ split off $\mathcal{L}\left(p_{1}^{2}, \ldots, p_{\delta}^{2}\right)$. If $R_{1}$ is in the fixed locus, then by symmetry, also $R_{2}$ is in the fixed locus. So, suppose by contradiction that $R_{1}, R_{2}$ are in the fixed locus. Then, after removing them from $\mathcal{L}\left(p_{1}^{2}, \ldots, p_{\delta}^{2}\right)$ we would remain with $\mathcal{L}^{\prime}$, the pull-back to $X$ of $\mathcal{L}_{n-2}\left(p^{n-6}, p_{1}^{2}, \ldots, p_{\delta}^{2}\right)$, which, by Claim 3.2 , has dimension $2 h-1-\varepsilon$. Hence we would have

$$
2 h-1-\varepsilon=\operatorname{dim}\left(\mathcal{L}_{n-2}\left(p^{n-6}, p_{1}^{2}, \ldots, p_{\delta}^{2}\right)\right)=\operatorname{dim}\left(\mathcal{L}\left(p_{1}^{2}, \ldots, p_{\delta}^{2}\right)\right) \geqslant 2 h+1-\varepsilon,
$$

a contradiction.

Let now $C$ be a general curve in $\mathcal{L}\left(p_{1}^{2}, \ldots, p_{\delta}^{2}\right)$. The above argument implies that no component of $C$ is a fixed curve of the anticanonical system of $X$. Then for any irreducible component $C^{\prime}$ of $C$ one has $K_{X} \cdot C^{\prime}<0$. In conclusion, $\mathcal{L}$ verifies the hypotheses (i)-(iv) of Theorem 2.3, and Claim 3.3 follows by the latter theorem.

We now end the proof of Proposition 3.1. Consider the locally closed family of curves

$$
\mathcal{G}:=\bigcup_{p_{1}, \ldots, p_{\delta}, r_{1}, r_{2}, q_{11}, q_{12}, q_{21}, q_{22}} \mathcal{L}_{n}\left(p^{n-4}, p_{1}^{2}, \ldots, p_{\delta}^{2} ;\left[q_{11}, q_{12}, q_{21}, q_{22}\right]\right)
$$

where the union is made by varying $p_{1}, \ldots, p_{\delta}$ among all $\delta$-tuples of general distinct points of $X, r_{1}, r_{2}$ among all pairs of distinct lines through $p$ and $q_{i j} \neq p$ among all pairs of distinct points of $r_{i}$, for $1 \leqslant i, j \leqslant 2$.

Of course $\mathcal{G}$ is irreducible and rational, and we have a map $\alpha: \mathcal{G} \rightarrow-\rightarrow \mathcal{R}_{g}^{0}$ which sends a general curve $\Gamma \in \mathcal{G}$ to $(C, \eta)$, where $C$ is the normalization of $\Gamma$, and $\eta=$ $\mathcal{O}_{C}\left(q_{11}+q_{12}-q_{21}-q_{22}\right)$, where, by abusing notation, we denote by $q_{i j}$ their inverse images in $C$, for $1 \leqslant i, j \leqslant 2$. Since $\mathcal{H} \rightarrow \rightarrow \mathcal{M}_{g, 4}^{1}$ is dominant by $[1, \S 5]$, then $\alpha$ is also dominant by Lemma 2.1. This proves the proposition.

\section{Dimension of $\mathcal{R}_{g}^{0}$}

In this section we finish the proof of Theorem 1.1 with the:

Proposition 4.1. The irreducible locus $\mathcal{R}_{g}^{0}$ has dimension $2 g+1$ if $g \geqslant 5$. 
Proof. Let $\mathcal{H}_{g, 4}$ denote the Hurwitz scheme parametrizing isomorphism classes of genus $g$ degree 4 covers of $\mathbb{P}^{1}$. We have a commutative diagram

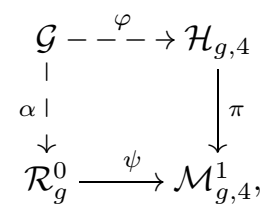

where $\pi$ and $\psi$ are the forgetful maps, $\alpha$ is the dominant map from the last part of the proof of Proposition 3.1 and $\varphi$ maps a general curve $\Gamma \in \mathcal{G}$ to the degree 4 cover defined by $2\left(q_{11}+q_{12}\right) \sim 2\left(q_{21}+q_{22}\right)$, using the notation of the proof of Proposition 3.1. Note that $\psi$ is finite, whence the dimension of $\mathcal{R}_{g}^{0}$ equals the dimension of the image of $\pi \circ \varphi$.

The image of $\varphi$ coincides with the locus $\mathcal{D} \subset \mathcal{H}_{g, 4}$ parametrizing covers with two pairs of distinct ramification points each over the same branch point. By Riemann's existence theorem, $\mathcal{D}$ has codimension 2 in $\mathcal{H}_{g, 4}($ whence $\operatorname{dim}(\mathcal{D})=2 g+1)$. Since $\mathcal{G}$ is irreducible (cf. the proof of Proposition 3.1), so is $\mathcal{D}$. Moreover, as the bielliptic locus in $\mathcal{M}_{g}$ has dimension $2 g-2$ and each bielliptic curve has a one-dimensional family of $g_{4}^{1} \mathrm{~s}$, the locus in $\mathcal{H}_{g, 4}$ with bielliptic domain curve has dimension $2 g-1$. Thus, the general element in the image of $\pi \circ \varphi$ is not bielliptic, whence the general element $(C, \eta)$ in the image of $\alpha$ has Prym-canonical image birational to $C$, by Corollary 2.2 (and necessarily singular, by Lemma 2.1(ii)). It follows that the fibre over $C$ of the restriction of $\pi$ to $\mathcal{D}$ is finite. Indeed, $C$ has finitely many preimages $(C, \eta)$ in the image of $\alpha$, and the Prym-canonical model of each of those has finitely many singular points, determining by Lemma 2.1(ii) only finitely many covers in $\mathcal{D} \subset \mathcal{H}_{g, 4}$ mapping to $C$ by $\pi$. Thus, the restriction of $\pi$ to $\mathcal{D}$ is generically finite, whence the image of $\pi \circ \varphi$ has dimension $2 g+1$.

\section{Proof of Proposition 1.2 and some examples}

Consider again the locus $\mathcal{D} \subset \mathcal{H}_{g, 4}$ from the proof of Proposition 4.1 parametrizing isomorphism classes of covers with two pairs of distinct ramification points each over a single branch point. By Riemann's existence theorem again, the general point in $\mathcal{D}$ corresponds to a cover with only two such branch points. By Lemma 2.1 (ii), if the domain curve has only one $g_{4}^{1}$, which is automatic if $g \geqslant 10$, then the Prym-canonical model of such a curve has precisely two nodes. It cannot have fewer singularities by Lemma 2.1. Thus, Proposition 1.2 is proved for $g \geqslant 10$.

Instead of embarking in a more refined treatment for $g \leqslant 9$, we note that certain curves on Enriques surfaces provide examples, for any genus $g \geqslant 5$, of curves with two-nodal Prym-canonical models, thus finishing the proof of Proposition 1.2: 
Example 5.1. The general Enriques surface $S$ contains no smooth rational curves [3] and contains smooth elliptic curves $E_{1}, E_{2}, E_{3}$ with $E_{i} \cdot E_{j}=1$ for $i \neq j$ (and $E_{i}^{2}=0$ by adjunction), for $1 \leqslant i, j \leqslant 3$, cf. e.g. [7, Thm. 3.2] or [8, IV.9.E, p. 273]. It also contains a smooth elliptic curve $E_{1,2}$ such that $E_{1,2} \cdot E_{1}=E_{1,2} \cdot E_{2}=2$, and $E_{1,2} \cdot E_{3}=1$, cf. e.g. [7, Thm. 3.2] or [8, IV.9.B, p. 270]. In particular, none of the numerical equivalence classes of $E_{1}, E_{2}, E_{3}, E_{1,2}$ are divisible in $\operatorname{Num}(S)$.

Consider, for any $g \geqslant 5$, the line bundle

$$
H_{g}:= \begin{cases}\mathcal{O}_{S}\left(\frac{g-2}{2} E_{1}+E_{2}+E_{3}\right), & g \text { even } \\ \mathcal{O}_{S}\left(\frac{g-1}{2} E_{1}+E_{1,2}\right), & g \text { odd } .\end{cases}
$$

The absence of smooth rational curves yields that $H_{g}$ is nef. As $H_{g}^{2}=2 g-2$, all curves in $\left|H_{g}\right|$ have arithmetic genus $g$. Moreover, we claim that $\phi\left(H_{g}\right)=E_{1} \cdot H_{g}=$ 2 (see the introduction for the definition of $\phi$ ) and that the only numerical class computing $\phi\left(H_{g}\right)$ is $E_{1}$. Indeed, if $g$ is even (resp., odd), then $E_{1} \cdot H_{g}=2, E_{2} \cdot H_{g}=$ $E_{3} \cdot H_{g}=\frac{g}{2} \geqslant 3$ (resp., $E_{1} \cdot H_{g}=2, E_{1,2} \cdot H_{g}=g-1 \geqslant 4$ ), and if $E$ is any nonzero effective divisor not numerically equivalent to any of $E_{1}, E_{2}, E_{3}$ (resp., $E_{1}, E_{1,2}$ ), then $E \cdot E_{1}>$ $0, E \cdot E_{2}>0$ and $E \cdot E_{3}>0$ (resp., $E \cdot E_{1}>0$ and $E \cdot E_{1,2}>0$ ) by [10, Lemma 2.1], so that $E \cdot H_{g} \geqslant \frac{g-2}{2}+2=\frac{g}{2}+1 \geqslant 4$ (resp., $E \cdot H_{g} \geqslant \frac{g-1}{2}+1=\frac{g+1}{2} \geqslant 3$ ).

By [8, Prop. 4.5.1, Thm. 4.6.3, Prop. 4.7.1, Thm. 4.7.1] the complete linear system $\left|H_{g}\right|$ is therefore base point free and defines a morphism $\varphi_{H_{g}}$ that is birational onto a surface with only double lines as singularities; the double lines are the images of curves computing $\phi\left(H_{g}\right)$, which, by what we said above, are $E_{1}$ and $E_{1}^{\prime}$, the only member of $\left|E_{1}+K_{S}\right|$. Thus, the image of $\varphi_{H_{g}}$ is a surface with precisely two double lines $\varphi_{H_{g}}\left(E_{1}\right)$ and $\varphi_{H_{g}}\left(E_{1}^{\prime}\right)$ as singularities. Therefore, $\varphi_{H_{g}}$ maps a general smooth $C \in|H|$ to a curve with precisely two nodes. Since $\varphi_{H_{g}}$ restricted to $C$ is the Prym-canonical map associated to $\eta:=\mathcal{O}_{C}\left(K_{S}\right)$ by [8, Cor. 4.1.2], a general smooth curve $C$ in $\left|H_{g}\right|$ together with $\eta$ is an example of a Prym curve of any genus $g \geqslant 5$ with two-nodal Prym-canonical model.

We prove in $\left[6\right.$, Thm. 2] that the general element in $\mathcal{R}_{g}^{0}$ is obtained in this way precisely for $5 \leqslant g \leqslant 8$.

Similar examples for odd $g \geqslant 7$ are obtained from the line bundle $H_{g}:=$ $\mathcal{O}_{S}\left(\frac{g-1}{2} E_{1}+2 E_{2}\right)$ or $H_{g}:=\mathcal{O}_{S}\left(\frac{g-1}{2} E_{1}+2 E_{2}+K_{S}\right)$, but (again by [6, Thm. 2]) the general element in $\mathcal{R}_{g}^{0}$ is not obtained in this way.

We conclude with an example of curves of genus 5 on an Enriques surface with 4-nodal Prym-canonical models and a result that will be used in [6]:

Example 5.2. With the same notation as in the previous example, set $H:=$ $\mathcal{O}_{S}\left(2 E_{1}+2 E_{2}+K_{S}\right)$. Then $H^{2}=8$, so that any curve in $|H|$ has arithmetic genus 5 . Moreover, $\phi(H)=2$ and one easily checks that $E_{1}$ and $E_{2}$ are the only numerical 
equivalence classes computing $\phi(H)$. As in the previous example, the complete linear system $|H|$ is base point free and defines a morphism $\varphi_{H}$ that is birational onto a surface with precisely four double lines as singularities, namely the images of $E_{1}, E_{2}, E_{1}^{\prime}$ and $E_{2}^{\prime}$, where $E_{i}^{\prime}$ is the only member of $\left|E_{i}+K_{S}\right|, i=1,2$. Thus $\varphi_{H}$ maps a general smooth $C \in|H|$ to a curve with precisely four nodes, so that, again by [8, Cor. 4.1.2], the pairs $\left(C, \mathcal{O}_{C}\left(K_{S}\right)\right)$ are genus 5 Prym curves with 4-nodal Prym-canonical models.

Also note that for any smooth $C \in|H|$, we have

$$
\omega_{C} \simeq \mathcal{O}_{C}\left(E_{1}+E_{2}\right)^{\otimes 2} \simeq \mathcal{O}_{C}\left(E_{1}+E_{2}+K_{S}\right)^{\otimes 2},
$$

whence $C$ has two autoresidual $g_{4}^{1} \mathrm{~s}$, namely $\left|\mathcal{O}_{C}\left(E_{1}+E_{2}\right)\right|$ and $\left|\mathcal{O}_{C}\left(E_{1}+E_{2}+K_{S}\right)\right|$, and their difference is $\mathcal{O}_{C}\left(K_{S}\right)$. (A complete linear system $|D|$ is called autoresidual if $D$ is a theta-characteristic, that is, $2 D \sim \omega_{D}$.) Thus, $\left(C, \mathcal{O}_{C}\left(K_{S}\right)\right)$ belongs to the locus in $\mathcal{R}_{5}$ consisting of Prym curves $(C, \eta)$ carrying a theta-characteristic $\theta$ such that $h^{0}(\theta)=h^{0}(\theta+\eta)=2$. The next result shows that this is a general phenomenon in $\mathcal{R}_{5}^{0}$.

Proposition 5.3. The locus in $\mathcal{R}_{5}^{0}$ of curves with 4-nodal Prym-canonical model is an irreducible unirational divisor whose closure in $\mathcal{R}_{5}$ coincides with the closure of the locus of Prym curves $(C, \eta)$ carrying a theta-characteristic $\theta$ with $h^{0}(\theta)=h^{0}(\theta+\eta)=2$.

Proof. Let us denote by $\mathcal{D}_{5}^{0}$ the locus of curves in $\mathcal{R}_{5}^{0}$ with 4-nodal Prymcanonical model, which is nonempty by the previous example. Let $\mathcal{V}$ denote the locus of curves of type $(4,4)$ on $\mathbb{P}^{1} \times \mathbb{P}^{1}$ with 4 nodes lying on the 4 nodes of a "square" configuration of two fibres of each projection to $\mathbb{P}^{1}$. We will prove that $\mathcal{V}$ is irreducible of dimension 16 and that there is a birational morphism

$$
f: \mathcal{D}_{5}^{0} \longrightarrow \mathcal{V}^{\prime}:=\mathcal{V} / \operatorname{Aut}\left(\mathbb{P}^{1} \times \mathbb{P}^{1}\right) .
$$

To define $f$, let $(C, \eta) \in \mathcal{D}_{5}^{0}$. By Lemma 2.1 there are four pairs of distinct points $(p, q),(x, y),\left(p^{\prime}, q^{\prime}\right)$ and $\left(x^{\prime}, y^{\prime}\right)$ on $C$, each identified by the Prym-canonical $\operatorname{map} \varphi: C \rightarrow \mathbb{P}^{3}$, such that

$$
\begin{aligned}
2(p+q) \sim 2(x+y), \quad 2\left(p^{\prime}+q^{\prime}\right) & \sim 2\left(x^{\prime}+y^{\prime}\right) \quad \text { and } \\
\eta \sim p+q-x-y & \sim x^{\prime}+y^{\prime}-p^{\prime}-q^{\prime} .
\end{aligned}
$$

In particular, we get that

$$
p+q+p^{\prime}+q^{\prime} \sim x+y+x^{\prime}+y^{\prime},
$$

thus defining a base point free $g_{4}^{1}$ on $C$, which we call $\ell_{1}$. We let $\mathcal{L}_{1}$ on $C$ be the corresponding line bundle. Since there exists a pencil of hyperplanes in $\mathbb{P}^{3}$ through 
any two of the four nodes of $\Gamma:=\varphi(C)$, we see that

$$
h^{0}\left(\omega_{C}(\eta)-\mathcal{L}_{1}\right)=h^{0}\left(\omega_{C}(\eta)\left(-p-q-p^{\prime}-q^{\prime}\right)\right)=2 .
$$

We claim that

$$
h^{0}\left(\omega_{C}(\eta)-2 \mathcal{L}_{1}\right)=0
$$

Indeed, if not, we would have $\omega_{C}(\eta) \simeq 2 \mathcal{L}_{1}$, which together with (4) would yield that $\Gamma \subset \mathbb{P}^{3}$ is contained in a quadric cone $Q$, with the pullback of the ruling of the cone cutting $\ell_{1}$ on $C$. Let $\widetilde{Q}$ be the desingularization of $Q$. Then $\widetilde{Q} \simeq \mathbb{F}_{2}$. Since $\ell_{1}$ is base point free, $\Gamma$ does not pass through the vertex of $Q$, so that we may consider $\Gamma$ as a curve in $\widetilde{Q}$. Denote by $\sigma$ the minimal section of $\mathbb{F}_{2}$ (thus, $\sigma^{2}=-2$ ), which is contracted to the vertex of $Q$, and by $\mathfrak{f}$ the class of the fibre of the ruling. Then, since $\Gamma \cdot \mathfrak{f}=4$ and $\Gamma \cdot \sigma=0$, we get that $\Gamma \sim 4 \sigma+8 \mathfrak{f}$. In particular, $\left.\omega_{\Gamma} \simeq \mathcal{O}_{\Gamma}\left(K_{\widetilde{Q}}+\Gamma\right)\right) \simeq \mathcal{O}_{\Gamma}(2 \sigma+4 \mathfrak{f}) \simeq \mathcal{O}_{\Gamma}(4 \mathfrak{f})$. Thus, from (3) we obtain

$$
\omega_{C} \simeq \varphi^{*}\left(\omega_{\Gamma}\right)\left(-p-q-x-y-p^{\prime}-q^{\prime}-x^{\prime}-y^{\prime}\right) \simeq \mathcal{O}_{C}\left(4 \mathcal{L}_{1}-2 \mathcal{L}_{1}\right) \simeq \mathcal{O}_{C}\left(2 \mathcal{L}_{1}\right),
$$

yielding $\eta=0$, a contradiction. This proves (5).

The relations (4) and (5) imply that $\Gamma \subset \mathbb{P}^{3}$ is contained in a smooth quadric surface $Q \simeq \mathbb{P}^{1} \times \mathbb{P}^{1}$. The first ruling is defined by the pencil $\ell_{1}$, whereas the second is defined by the pencil $\ell_{2}=\left|\mathcal{L}_{2}\right|$, where $\mathcal{L}_{2}:=\omega_{C}(\eta)-\mathcal{L}_{1}=\omega_{C}(\eta)\left(-p-q-p^{\prime}-q^{\prime}\right)$ by $(4)$. The curve $\Gamma$ is of type $(4,4)$ on $Q$, with four nodes. Since $\omega_{\Gamma} \simeq \omega_{\mathbb{P}^{1} \times \mathbb{P}^{1}}(C) \simeq \mathcal{O}_{\Gamma}(2,2)$, we see that $\varphi^{*}\left(\omega_{\Gamma}\right) \simeq\left(\omega_{C}(\eta)\right)^{\otimes 2} \simeq \omega_{C}^{\otimes 2}$. Thus,

$$
\omega_{C} \simeq \omega_{C}^{\otimes 2}\left(-p-q-x-y-p^{\prime}-q^{\prime}-x^{\prime}-y^{\prime}\right)
$$

whence

$$
\omega_{C} \simeq \mathcal{O}_{C}\left(p+q+x+y+p^{\prime}+q^{\prime}+x^{\prime}+y^{\prime}\right)
$$

Combining with (2), we find that

$$
\mathcal{L}_{2} \simeq \omega_{C}(\eta)\left(-p-q-p^{\prime}-q^{\prime}\right) \simeq \mathcal{O}_{C}\left(p+q+x^{\prime}+y^{\prime}\right) \simeq \mathcal{O}_{C}\left(p^{\prime}+q^{\prime}+x+y\right) .
$$

The relations (3) and (7) tell us that the four nodes of $\Gamma$ lie on two pairs of fibres of each ruling of $\mathbb{P}^{1} \times \mathbb{P}^{1}$, thus showing that $\Gamma \in \mathcal{V}$. Of course this is all well-defined up to automorphisms of $\mathbb{P}^{1} \times \mathbb{P}^{1}$, so we see that the construction associates to $(C, \eta)$ an element in $\mathcal{V}^{\prime}$, which we define to be the image of $(C, \eta)$ by $f$.

This defines the map $f$, and in particular shows that $\mathcal{V}$ is nonempty. We also note for later use that $\omega_{C} \simeq 2 \mathcal{L}_{1} \simeq 2 \mathcal{L}_{2}$, so that $\mathcal{D}_{5}^{0}$ is contained in the locus of Prym curves $(C, \eta)$ carrying a theta-characteristic $\theta$ with $h^{0}(\theta)=h^{0}(\theta+\eta)=2$, which we henceforth call $T_{5}$. Moreover, via the forgetful map $\mathcal{R}_{5} \rightarrow \mathcal{M}_{5}$, the locus $T_{5}$ maps to the locus of curves with two (complete) autoresidual $g_{4}^{1} \mathrm{~s}$, which we call $\mathcal{B}_{5}$. 
We next prove that $\mathcal{V}$ is irreducible rational of dimension 16 .

For any $X \in \mathcal{V}$, let $\nu: C \rightarrow X$ be the normalization; $C$ has genus 5 . If $z_{i}, i=$ $1,2,3,4$, are the nodes of $X$, then the complete linear system

$$
\left|\mathcal{O}_{\mathbb{P}^{1} \times \mathbb{P}^{1}}(X) \otimes \mathcal{J}_{z_{1}}^{2} \otimes \mathcal{J}_{z_{2}}^{2} \otimes \mathcal{J}_{z_{3}}^{2} \otimes \mathcal{J}_{z_{4}}^{2}\right|
$$

has dimension 12, as expected. Indeed, letting $r$ be its dimension, we clearly have $r \geqslant 12$; on the other hand, this complete linear system induces a $g_{16}^{r-1}$ on $C$, whence $r-1 \leqslant 11$ by Riemann-Roch. It follows that $\mathcal{V}$ is birational to $\mathbb{P}^{12} \times\left(\operatorname{Sym}^{2}\left(\mathbb{P}^{1}\right)\right)^{2}$ (because of the freedom of varying the four lines in the square configuration), in particular it is irreducible rational of dimension $12+4=16$.

We now define the inverse of $f$. Given a curve $X \in \mathcal{V}$, let $\mathcal{L}_{1}$ and $\mathcal{L}_{2}$ be the line bundles of degree 4 on $C$ defined by the pullbacks of the two rulings on $\mathbb{P}^{1} \times \mathbb{P}^{1}$. By the special position of the 4 nodes of $X$, the four pairs of points $C$ lying above the four nodes of $X$, say $(p, q),(x, y),\left(p^{\prime}, q^{\prime}\right)$ and $\left(x^{\prime}, y^{\prime}\right)$, satisfy

$$
\begin{aligned}
& \mathcal{L}_{1} \simeq \mathcal{O}_{C}\left(p+q+p^{\prime}+q^{\prime}\right) \simeq \mathcal{O}_{C}\left(x+y+x^{\prime}+y^{\prime}\right), \\
& \mathcal{L}_{2} \simeq \mathcal{O}_{C}\left(p+q+x^{\prime}+y^{\prime}\right) \simeq \mathcal{O}_{C}\left(x+y+p^{\prime}+q^{\prime}\right),
\end{aligned}
$$

in particular, $\eta:=\mathcal{L}_{1}-\mathcal{L}_{2}$ is 2 -torsion. Moreover, one can easily verify that $\omega_{C}(\eta) \simeq$ $\mathcal{L}_{1}+\mathcal{L}_{2}$. Thus, the normalization $\nu: C \rightarrow X \subset \mathbb{P}^{1} \times \mathbb{P}^{1}$ followed by the embedding of $\mathbb{P}^{1} \times \mathbb{P}^{1}$ as a quadric in $\mathbb{P}^{3}$ induces the Prym-canonical map associated to $\omega_{C}(\eta)$, so that $(C, \eta)$ has a 4 -nodal Prym-canonical image. One readily checks that this map is the inverse of the map $f$ defined above. Thus, we have proved that $\mathcal{D}_{5}^{0}$ is irreducible of dimension $\operatorname{dim} \mathcal{V} /\left(\operatorname{Aut}\left(\mathbb{P}^{1} \times \mathbb{P}^{1}\right)\right)=16-6=10$.

We have left to prove that the closure of $\mathcal{D}_{5}^{0}$ in $\mathcal{R}_{5}$ coincides with the closure of $T_{5}$. We proved above that $\mathcal{D}_{5}^{0}$ is contained in $T_{5}$ and that the latter maps, via the finite forgetful map $\mathcal{R}_{5} \rightarrow \mathcal{M}_{5}$, to the locus $\mathcal{B}_{5}$ of curves with two autoresidual $g_{4}^{1} \mathrm{~s}$, which is irreducible of dimension 10 by [11, Thm. 2.10]. Below we give a direct proof of the latter fact, which also proves that the general member of $\mathcal{B}_{5}$ carries exactly two $g_{4}^{1} \mathrm{~s}$, equivalently two theta characteristics $\theta$ and $\theta^{\prime}$ such that $h^{0}(\theta)=h^{0}\left(\theta^{\prime}\right)=2$. It will follow that there is an inverse rational map $\mathcal{B}_{5}-\rightarrow T_{5}$ mapping $C$ to $\left(C, \theta-\theta^{\prime}\right)$, proving that also $T_{5}$ is irreducible of dimension 10. Its closure must therefore coincide with the closure of $\mathcal{D}_{5}^{0}$, finishing the proof of the proposition.

So let $C$ be a smooth, irreducible curve of genus 5 and consider its canonical embedding $C \subset \mathbb{P}^{4}$. Given $\xi=|D|$ a (complete) $g_{4}^{1}$ on $C$, the divisors in $\xi$ span planes which sweep out a quadric $Q_{\xi}$ of rank $r<5$. If $\xi$ is not autoresidual, then $Q_{\xi}$ has rank $r=4$ and it has another 1-dimensional system of planes which cut out on $C$ the divisors of $\xi^{\prime}=\left|K_{C}-D\right|$. In this case $Q_{\xi}=Q_{\xi^{\prime}}$. Hence $\xi$ is autoresidual if and only if $Q_{\xi}$ has rank 3 , and therefore it possesses only one 1-dimensional family of planes. This means that the homogeneous ideal of a curve in $\mathcal{B}_{5}$ in its canonical 
embedding contains two distinct rank 3 quadrics. Hence the general curve $C$ in $\mathcal{B}_{5}$ is obtained by intersecting two general rank 3 quadrics in $\mathbb{P}^{4}$ with another general quadric. Note that the two rank 3 quadrics cut out a Del Pezzo surface $S$ with 4 nodes, hence $C$ is a general quadric section on $S$. The two autoresidual $g_{4}^{1}$ on $C$ are cut out on $C$ by the conics of the two pencils on $S$ with base points two of the nodes.

From this description it follows that $\mathcal{B}_{5}$ is irreducible, 10-dimensional and that its general member contains precisely two autoresidual $g_{4}^{1} \mathrm{~s}$. Indeed, consider the $\mathbb{P}^{14}$ parametrizing all quadrics in $\mathbb{P}^{4}$. The locus $\mathcal{X}$ of quadrics of rank $r \leqslant 3$ is nondegenerate and has dimension 11 . The net of quadrics defining a general curve $C$ in $\mathcal{B}_{5}$ corresponds to a plane in $\mathbb{P}^{14}$ containing a general secant line to $\mathcal{X}$ (which, by its generality, contains only two points in $\mathcal{X}$ ), and an easy count of parameters shows that these planes clearly fill up a variety of dimension 34. Modding out by the 24-dimensional group of projective transformations of $\mathbb{P}^{4}$, we get dimension 10 for $\mathcal{B}_{5}$.

Remark 5.4. Denote, as in the last proof, by $\mathcal{D}_{0}^{5}$ the locus of Prym curves $(C, \eta)$ carrying a theta-characteristic $\theta$ with $h^{0}(\theta)=h^{0}(\theta+\eta)=2$. By [4, Prop. 7.3 and Thm. 7.4] the locus $\mathcal{D}_{0}^{5}$ maps, via the Prym map $\mathcal{P}_{5}: \mathcal{R}_{5} \rightarrow \mathcal{A}_{4}$, to the irreducible divisor $\theta_{\text {null }}$ of principally polarized abelian varieties whose theta-divisor has a singular point at a 2-torsion point, and moreover the general member of $\mathcal{P}_{5}\left(\mathcal{D}_{0}^{5}\right)$ has precisely one ordinary double point, cf. [4, Pf. of Prop. 7.5]. It would be interesting to know if $\mathcal{D}_{0}^{5}$ dominates $\theta_{\text {null }}$.

By [4, Prop. 7.3] one knows that the closure of $\mathcal{P}_{5}^{-1}\left(\theta_{\text {null }}\right)$ is the closure of the locus of Prym curves $(C, \eta)$ carrying a theta-characteristic $\theta$ such that $h^{0}(\theta)+$ $h^{0}(\theta+\eta)$ is even, which properly contains $\mathcal{D}_{0}^{5}$.

Remark 5.5. By contrast, if we consider the adjoint line bundle of the one in Example 5.2 , that is, $H^{\prime}:=\mathcal{O}_{S}\left(2 E_{1}+2 E_{2}\right)$, then by [8, Prop. 4.1.2, Thm. 4.7.1, (F) p. 277] the morphism $\varphi_{H^{\prime}}$ defined by $\left|H^{\prime}\right|$ is of degree 2 onto a quartic Del Pezzo surface. In particular, $\varphi_{H^{\prime}}$ maps any smooth $C \in|H|$ doubly onto an elliptic quartic curve in $\mathbb{P}^{3}$. Hence, the Prym curve $\left(C, \mathcal{O}_{C}\left(K_{S}\right)\right)$ belongs to the locus $\mathcal{R}_{5}^{0, n b}$ described in Corollary 2.2.

\section{References}

1. Arbarello, E. and Cornalba, M., Footnotes to a paper of Beniamino Segre, Math. Ann. 256 (1981), 341-362. MR0626954

2. Ballico, E. and Del Centina, A., Ramification points of double coverings of curves and Weierstrass points, Ann. Mat. Pura Appl. 177 (1999), 293313. MR1747636 
3. Barth, W. and Peters, C., Automorphisms of Enriques surfaces, Invent. Math. 73 (1983), 383-412. MR0718937

4. Beauville, A., Prym varieties and the Schottky problem, Invent. Math. 41 (1977), 149-196. MR0572974

5. Brodmann, M. and Schenzel, P., Arithmetic properties of projective varieties of almost minimal degree, J. Algebraic Geom. 16 (2007), 347-400. MR2274517

6. Ciliberto, C., Dedieu, T., Galati, C. and Knutsen, A. L., Moduli of curves on Enriques surfaces, Adv. Math. 365 (2020), 10.1016/j.aim. 2020. 107010. MR4065262

7. Cossec, F. R., On the Picard group of Enriques surfaces, Math. Ann. 271 (1985), 577-600. MR0790116

8. Cossec, F. R. and Dolgachev, I. V., Enriques Surfaces. I, Progress in Mathematics 76, Birkhäuser Boston, Inc., Boston, MA, 1989. MR0986969

9. Harris, J. and Morrison, I., Moduli of Curves, Graduate Text in Mathematics 187, Springer, 1998. MR1631825

10. Knutsen, A. L. and Lopez, A. F., A sharp vanishing theorem for line bundles on K3 or Enriques surfaces, Proc. Amer. Math. Soc. 135 (2007), 34953498. MR2336562

11. Knutsen, A. L., Lelli-Chiesa, M. and Verra, A., Moduli of non-standard Nikulin surfaces in low genus, Ann. Sc. Norm. Super. Pisa Cl. Sci., to appear. 10.2422/2036-2145.201802_018. arXiv: 1802 . 01201.

12. Lange, H. and Sernesi, E., Quadrics containing a Prym-canonical curve, J. Algebraic Geom. 5 (1996), 387-399. MR1374713

Ciro Ciliberto

Dipartimento di Matematica

Università di Roma Tor Vergata

Via della Ricerca Scientifica

IT-00173 Roma

Italy

cilibert@mat.uniroma2.it

Thomas Dedieu

Institut de Mathématiques de Toulouse UMR5219

Université de Toulouse — CNRS, UPS

IMT

F-31062

Toulouse Cedex 9

France

thomas.dedieu@math.univ-toulouse.fr

Received June 24, 2019

in revised form November 13, 2019
Concettina Galati

Dipartimento di Matematica e Informatica

Università della Calabria

via P. Bucci, cubo 31B

IT-87036 Arcavacata di Rende (CS)

Italy

galati@mat.unical.it

Andreas Leopold Knutsen

Department of Mathematics

University of Bergen

Postboks 7800

NO-5020 Bergen

Norway

andreas.knutsen@math.uib.no 